\title{
Temperature-dependent coercive field measured by a quantum dot strain
}

gauge

Yan Chen ${ }^{1}$, Yang Zhang ${ }^{1}$, Robert Keil ${ }^{1}$, Michael Zopf ${ }^{1}$, Fei Ding ${ }^{1,2}$ and Oliver G. Schmidt ${ }^{1,3}$

${ }^{I}$ Institute for Integrative Nanosciences, IFW Dresden, Helmholtzstraße 20, 01069 Dresden, Germany

${ }^{2}$ Institut für Festkörperphysik, Leibniz Universität Hannover, Appelstraße 2, 30167 Hannover, Germany

${ }^{3}$ Chemnitz University of Technology, Reichenhainerstraße 70, 09107 Chemnitz, Germany

Coercive fields of piezoelectric materials can be strongly influenced by environmental temperature. We investigate this influence using a hetero-structure consisting of a single crystal piezoelectric film and a quantum dots containing membrane. Applying electric field leads to a physical deformation of the piezoelectric film, thereby inducing strain in the quantum dots and thus modifying their optical properties. The wavelength of the quantum dot emission shows butterfly-like loops, from which the coercive fields are directly derived. The results suggest that coercive fields at cryogenic temperatures are strongly increased, yielding values several tens of times larger than those at room temperature. We adapt a theoretical model to fit the measured data with very high agreement. Our work provides an efficient framework for predicting the properties of ferroelectric materials and advocate their practical applications, especially at low temperatures.

Keywords: coercive field, low temperature, piezoelectricity, quantum dot, strain gauge

Correspondence should be addressed to Prof. Dr. Fei Ding (f.ding@fkp.uni-hannover.de) 
Ferroelectric materials attract research interest for both fundamental physics and a broad range of applications. In particular, relaxor ferroelectric lead magnesium niobate-lead titanate (1$\mathrm{x})[\mathrm{Pb}(\mathrm{Mg} 1 / 3 \mathrm{Nb} 2 / 3) \mathrm{O} 3]-\mathrm{x}[\mathrm{PbTiO} 3]$ (PMN-PT) single crystals with compositions near the morphotropic phase boundary (MPB) exhibit superior piezoelectric activity. ${ }^{1,} \quad 2$ Microelectromechanical systems (MEMS) incorporating these single crystals enable highly sensitive sensors and low-voltage driven actuators. ${ }^{3,4,5}$ They are also frequently used as a strain engineering platform for manipulating the properties of various kinds of materials, such as semiconductors, ${ }^{6,7}$ superconductors ${ }^{8}$ and magnetic materials ${ }^{9}$ due to the ability to generate a giant displacement in response to an applied voltage. Strain engineering using PMN-PT has become a very active research area. ${ }^{10,11}$ Recent developments in aerospace exploration, lunar lander and even quantum optics have pushed applications into the regime of cryogenic temperatures. ${ }^{12,13,14,}$ 15 Considering the increasing demands for wide temperature operation, it is important to understand the temperature-dependent properties of these materials.

The coercive field reflecting the ease of domain reversal is one of the most important characteristic parameters for ferroelectrics. ${ }^{16,17}$ The Landau-Ginzburg mean field theory predicts the intrinsic temperature-dependent coercive field ${ }^{18,19}$. However, the intrinsic coercive field is only observed in ultrathin films or nano-particles where nucleation is inhibited by finite-size effects ${ }^{20-23}$. In most experiments the measured values of coercive fields are much smaller than the intrinsic values due to localized nucleation of reversed domains. These domains grow and coalesce through domain wall (DW) motion. The observed dynamics of DW motion are affected by temperature and show various temperature dependencies ${ }^{24-27}$.

In this work, we probe the temperature-dependent coercive field of single crystal PMN-PT film with a new optical method. We use dry etching and die bonding techniques to fabricate hetero- 
structures consisting of single crystal PMN-PT films and quantum dots (QDs). The PMN-PT films are used as actuators and QDs as an ultra-sensitive strain gauge. When cooled down to cryogenic temperatures, PMN-PT behaves like a very hard piezoelectric material ${ }^{28,29,30}$, but still outperforms other piezoelectric materials. ${ }^{31,32,33}$ They can generate strain to modify QDs emission peaks under electric bias. Results show that the coercive field strongly increases at low temperatures reaching $105 \mathrm{kV} / \mathrm{cm}$ at 5 Kelvin, 70 times larger compared to room temperature values. The data is well fitted by an adapted model borrowed from the field of ferromagnetic materials.

Epitaxial QDs are of great interest in the field of quantum information processing as they can emit single photons ${ }^{34}$ and entangled photon pairs ${ }^{35}$. They are zero dimensional system formed by encapsulating a low band semiconductor material with a high band gap semiconductor material. Electrons in the QDs have discrete energetic levels due to the strong quantum confinement. And in this sense QDs are referred as 'artificial atom'. Recent studies suggest that QDs are very sensitive to external perturbations such as strain, electric or magnetic fields. ${ }^{28}$ For instance, single photons from dissimilar QDs can be set into spectral resonance, ${ }^{36,}{ }^{37}$ with the biaxial strain generated by PMN-PT. In turn, III-V semiconductor QDs can monitor the subtle deformation of PMN-PT as an ultra-sensitive strain gauge. The cryogenic strain gauge is essential to investigate the properties of piezoelectric materials. Compared with commercial foil-type strain gauges, QDs are particularly suitable for cryogenic measurement purposes. Their size is much reduced while maintaining a very high sensitivity. ${ }^{38}$

Figure 1a is the artistic sketch of our device. The piezoelectric actuator mainly consists of a PMNPT film. A gold layer was sputtered on the backside as a gate electrode. The PMN-PT film is bonded onto a silicon substrate with epoxy glue. Top electrodes were subsequently fabricated by photolithography and metal deposition. To make patterns the film, we applied focused ion beam. 
The milled trenches are deep enough to penetrate into the silicon substrate. Afterwards, the device was undercut by wet chemical etching to form suspended PMN-PT cantilevers in order to reduce clamping from the substrate. Then a QDs containing nano-membrane was transferred onto the single crystal PMN-PT film actuators by a flip-chip bonder. Under electric bias, the actuator is deformed physically and introduces strain to the QDs, leading to spectral shifts of the emission peaks. Figure $1 \mathrm{~b}$ shows the final device (false color). The blue structure is the QDs containing nano-membrane and its structure is sketched in the lower panel. The opposing pair of cantilevers is always subjected to the same voltage, on one hand, to keep the displacement in the nanomembrane small and on the other hand to increase the strain in the QD membrane and therefore get higher sensitivity to measure the coercive field more precise. It is worth noting that our special MEMS design here is capable of delivering very large strain values. Below is a simplified sketch of our device. The strain in the QD film is amplified by a factor of $4 \mathrm{~L} / \mathrm{d}$, where $\mathrm{L}$ is the length of each actuation leg and $\mathrm{d}$ is the gap width. This improves the result and extends its application potential significantly.

In the next step, we utilize strain generated by the suspended PMN-PT film to shift the QDs emission peaks. The device was loaded in a cooled cryostat. Micro-photoluminescence ( $\mu$-PL) spectroscopy measurements as shown in Figure 2a were performed. During the cooling process, there is strain accumulated in the QD film due to the thermal expansion mismatch. However, the strain is not sufficiently large to induce bending or cracking of the QD film. The QD film is very robust and still survives under large strain. The QDs are excited by a c.w. laser at $532 \mathrm{~nm}$. Luminescence signal is collected with an objective and sent to a spectrometer for analysis. A computer-controlled source meter applies voltages to the PMN-PT actuator. The four actuation legs, sharing the same bottom electrode on the backside of the piezo film, work independently. 
Once we apply a voltage to the electrodes, these four actuation legs stretch or contract them inplane. The QD layer glued to the free ends of the actuators will be stretched or compresses correspondingly. Figure $2 \mathrm{~b}$ shows a typical $\mu$-PL spectrum of one GaAs QD. Inset shows the energetic levels of the QD. When varying the voltage on the PMN-PT film, emission peaks are recorded. Small electric fields (below the coercive field) shift the emission peaks from the QDs linearly (step A). With increasing the electric field beyond the coercive field, the emission peaks shift reversely as shown in Figure 2c (step B), indicating the strain jumps back at a high electric field. This phenomena is closely related with the coercive filed and is explained in the following. The strain behaviour of PMN-PT is dependent on its crystal structure. ${ }^{1}$ Our sample of PMN-PT has a PT-content of $28 \%$. The most plausible unit cell structure is rhombohedral. ${ }^{29,}{ }^{30}$ Further structural and compositional characterization of the PMN-PT thin film was performed by X-ray diffraction (XRD) with Co-K $\alpha$ radiation. The result is shown in Figure 3a. Only $(00 l)$ diffraction peaks of a pure perovskite phase of PMN-PT are displayed, confirming the micro-processing does not introduce any secondary phase or impurities. In PMN-PT with a unit cell surface orientation of [001], there are eight possible poling directions as shown Figure 3b. Without electric poling, polarizations in eight directions exist in equal numbers. To enhance the piezoelectric properties of PMN-PT, a very strong electric field needs to be applied in one direction, realigning some of the polarization directions so that they all work together in one direction. However, poling does not mean only one direction is left. In this study, the PMN-PT is fully poled along the [001] direction. The polarizations are aligned to [111], [111], [111] and [111] as is shown in Figure 3c.

The electric field is applied across the piezo film. There is virtually no electric field on the QDs. And according to semiconductor theory, QDs can only be affected by electric field when the QDs are embedded in an n-i-p structure. In that case, the top and bottom AlGaAs layers which sandwich 
the QDs are positively and negatively doped, respectively. The energy band can be tilted by a vertical external electric field then. In this work, the QD sample is not doped at all. And no electric field is applied to the QDs either. Therefore, the external electric field effect on the QDs can be excluded.

When decreasing electric field in [001] direction, net polarizations in [001] direction decrease gradually, resulting in a linearly increasing strain in the (001) plane due to relaxation of the lattice distortion (Figure 3d). And the QD emission peak shifts linearly as indicated by step A in Figure 2c. At the coercive field, there is no net polarization in the [001] direction (Figure 3e). The unit cell distortion disappears at this point. And strain in the (001) plane is maximized. When further decreasing the electric field, the polarization starts to flip to [001] and the unit cell starts to distort again (Figure 3f). Net polarizations in [001] keep increasing. Strain, thus the emission peak shifts back as is marked step B in Figure 2c. When sweeping voltages back, the emission peak undergoes similar behaviour marked by step C and step D. The configurations of polarizations under different voltages are sketched in Figure 3c-g. The emission peak against the electric field appears as a butterfly-like loop. The shape and size the device, including the ferroelectric film and the QDs have no influence on the results. The coercive field only reflects the property of the ferroelectric film itself and has nothing to do with the device shape or the strain gauge used. The shape and size influence the magnitude of the induced strain i.e. the shift of the QD emission peaks. But the 'strain turning point' does not change. We measured the butterfly-like loops under various temperatures. As there are slight asymmetries in some of these loops, the value of the coercive field is determined as the mean absolute value of positive and negative coercive fields. Coercive fields decrease very fast with rising temperature as shown in Figure 4a. 
The coercive field at 5 Kelvin is measured to be more than $100 \mathrm{kV} / \mathrm{cm}$. At room temperature, it drops to $1.5 \mathrm{kV} / \mathrm{cm}$. The coercive fields at various temperatures are plotted Figure $4 \mathrm{~b}$. The line width of QD photoluminescence increases as the temperature increases. Above 70 Kelvin, the QD photoluminescence signal becomes very weak. Nevertheless, the QD hosting material, i.e. the AlGaAs film itself can emit luminescence even at room temperature. We still can observe the their emission shifting induced by piezo strain. Another alternative could be to monitor the Raman peak of the AlGaAs. Therefore, using QD film to measure the coercive fields of piezoelectric materials can be done over a broad temperature range. To see how the coercive field changes over a wide temperature range, we also retrieved the data from Ref. [33] measured above 100 Kelvin. We stress that the thickness of the PMN-PT does not play a role in the determination of the coercive field. We have fabricated another device using a PMN-PT film of different thickness. The coercive field measured at 5 Kelvin agrees excellently with our present data.

The polarization in a ferroelectric material can be reversibly switched by an external magnetic field..$^{39,40}$ For instance, under an external triangle-wave magnetic field, the DWs undergo several dynamic steps, changing from relaxation to creep and from creep to flow. ${ }^{41-43}$ The crossover fields between these steps are denoted as $\mathrm{H} 1$ and $\mathrm{H} 2$. According to the theory of Nattermann et al. $\mathrm{H} 1$ and $\mathrm{H} 2$ can be written as ${ }^{43,44}$

$H_{1}=H_{C 0}\left[\left(U / k_{B} T \varphi\right)\left(1-H_{1} / H_{C 0}\right)^{\delta}\right]^{1 / \mu}$

$H_{2}=H_{C 0}\left[\left(U / k_{B} T\right)\left(1-H_{1} / H_{C 0}\right)^{\delta}\right]^{1 / \mu}$

where $\mathrm{U}, H_{C 0}$ are the characteristic pinning energy, and the critical de-pinning threshold field at $\mathrm{T}$ $=0$ Kelvin, respectively. $\mu$ is the dynamical exponent reflecting the nature of defects. ${ }^{23,40,41,45} k_{B}$ 
is the Boltzmann's constant. $\delta$ is dynamical exponent related to electric field dependence of energy barrier.

Due to the similarity of the underlying physics, the analysis can be extended to piezoelectric materials. By replacing magnetic field with electric field, we get the crossover fields E1 and E2 of piezoelectric materials. And coercive field can be well defined as $(E 1+E 2) / 2$ provided the frequency of the driven field is low ${ }^{43}$. Ec can be written as

$E_{c}=E_{C 0}\left[\left(U / k_{B} T\right)(1 / \varphi+1)\left(1-E_{1} / E_{C 0}\right)^{\delta}\right]^{1 / \mu}$

We take values $\mu=0.5$ and $\delta=2$ as in literatures. ${ }^{41,43,46}$ and do the theoretical fitting with formula (3). The fitted curve is marked by the red solid line in Figure $4 \mathrm{~b}$, which agrees very well with the measured data.

To summarize, we have developed a new optical method to measure the coercive field at cryogenic temperatures and find that cryogenic temperatures increase the coercive fields of PMN-PT dramatically, reaching $105 \mathrm{KV} / \mathrm{cm}$ at $5 \mathrm{Kelvin}$. The hetero-structure device fabricated here operates under low voltages and determines the properties of PMN-PT precisely. Our results are repeatable not only for the same device but also repeatable for a second device which has a different piezo film thickness. On one hand, the high quality single crystal piezoelectric film reduces the operation voltages, especially at cryogenic temperatures. On the other hand, QDs can feel subtle strain and change their emission peaks pronouncedly. We observe butterfly-like loops of the QDs emission peaks, a signature of coercivity. Our findings provide guidance for PMN-PTbased cryogenic applications such as non-volatile memory, ultrasound sensors and transducers. 


\section{ACKNOWLEDGEMENT}

The work was financially supported by the ERC Starting Grant No. 715770 (QD-NOMS), the BMBF Q.Com-H (16KIS0106) and the European Union Seventh Framework Programme 209 (FP7/2007-2013) under Grant Agreement No. 601126210 (HANAS). F.D. acknowledges the generous support of IFW Excellence Program. We thank B. Eichler, R. Engelhard, M. Bauer, S. Nestler and S. Harazim for technical supports. Y.C. would like to thank Dr. Zhao Dong at Max Planck Institute for Solid State Research for fruitful discussions. 


\section{References}

1. Baek, S.-H.; Rzchowski, M. S.; Aksyuk, V. A. Mrs Bull. 2012, 37, 1022-1029.

2. $\quad$ Chen, Y.; Zhang, Y.; Zhang, L.; Ding, F.; Schmidt, O. G. Nano Energy 2017, 31, 239-246.

3. Bellaiche, L.; Vanderbilt, D. Phys. Rev. Lett. 1999, 83, 1347-1350.

4. Yin, Z.-W.; Luo, H.-S.; Wang, P.-C.; Xu, G.-S. Ferroelectrics 1999, 229, 207-216.

5. $\quad$ Chen, Y.; Zhang, Y.; Karnaushenko, D.; Chen, L.; Hao, J.; Ding, F.; Schmidt, O. G. Adv.

Mater. 2017, 29, 1605165.

6. Minamisawa, R. A.; Süess, M. J.; Spolenak, R.; Faist, J.; David, C.; Gobrecht, J.; Bourdelle, K. K.; Sigg, H. Nat. Commun. 2012, 3, 1096.

7. Chu, M.; Sun, Y.; Aghoram, U.; Thompson, E. S. Annu. Rev. Mater. Res. 2009, 39, 203229.

8. Locquet, J. P.; Perret, J.; Fompeyrine, J.; Machler, E.; Seo, J. W.; Van Tendeloo, G. Nature 1998, 394, 453-456.

9. $\quad$ Wang, Z.; Wang, Y.; Luo, H.; Li, J.; Viehland, D. Phys. Rev. B 2014, 90, (13), 134103.

10. Chu, M.; Sun, Y.; Aghoram, U.; Thompson, E. S. Annu. Rev. Mater. Res. 2009, 39, 203229.

11. Schlom, G. D.; Chen, L.-Q.; Eom, C.-B.; Rabe, M. K.; Streiffer, K. S.; Triscone, J.-M. Annu. Rev. Mater. Res. 2007, 37, 589-626.

12. Jiang, X.; Rehrig, P. W.; Hackenberger, W. S.; Shrout, T. R. AIP Conf. Proc. 2006, 823, 1783-1789.

13. Xu, T.-B.; Tolliver, L.; Jiang, X.; Su, J. Appl. Phys. Lett. 2013, 102, 042906.

14. Chen, Y.; Zadeh, I. E.; Jöns, K. D.; Fognini, A.; Reimer, M. E.; Zhang, J.; Dalacu, D.; Poole, P. J.; Ding, F.; Zwiller, V.; Schmidt, O. G. Appl. Phys. Lett. 2016, 108, 182103.

15. Ding, F.; Singh, R.; Plumhof, J. D.; Zander, T.; Křápek, V.; Chen, Y. H.; Benyoucef, M.; Zwiller, V.; Dörr, K.; Bester, G.; Rastelli, A.; Schmidt, O. G. Phys. Rev. Lett. 2010, 104, 067405. 16. Bur, A.; Wong, K.; Zhao, P.; Lynch, C. S.; Amiri, P. K.; Wang, K. L.; Carman, G. P. Appl. Phys. Lett. 2011, 98, 262504.

17. Hu, J.-M.; Li, Z.; Chen, L.-Q.; Nan, C.-W. Nat. Commun. 2011, 2, 553.

18. V.L. Ginzburg, Z. E. Teor. Fiz 1945, 15, 739.

19. Fridkin, V. M.; Ducharme, S. Phys. Solid State 2001, 43, 1320-1324.

20. Ducharme, S.; Fridkin, V. M.; Bune, A. V.; Palto, S. P.; Blinov, L. M.; Petukhova, N. N.; Yudin, S. G. Phys. Rev. Lett. 2000, 84, 175-178.

21. Maksymovych, P.; Jesse, S.; Huijben, M.; Ramesh, R.; Morozovska, A.; Choudhury, S.; Chen, L.-Q.; Baddorf, A. P.; Kalinin, S. V. Phys. Rev. Lett. 2009, 102, 017601.

22. Highland, M. J.; Fister, T. T.; Richard, M.-I.; Fong, D. D.; Fuoss, P. H.; Thompson, C.; Eastman, J. A.; Streiffer, S. K.; Stephenson, G. B. Phys. Rev. Lett. 2010, 105, 167601.

23. Liu, S.; Grinberg, I.; Rappe, A. M. Nature 2016, 534, 360-363.

24. Ma, B.; Hu, Z.; Liu, S.; Narayanan, M.; Balachandran, U. Appl. Phys. Lett. 2013, 102, 072901.

25. Mendonça, E. C.; Jesus, C. B. R.; Folly, W. S. D.; Meneses, C. T.; Duque, J. G. S.; Coelho, A. A. J. Appl. Phys. 2012, 111, 053917.

26. Suess, D.; Breth, L.; Lee, J.; Fuger, M.; Vogler, C.; Bruckner, F.; Bergmair, B.; Huber, T.; Fidler, J.; Schrefl, T. Phys. Rev. B 2011, 84, 224421. 
27. Maaz, K.; Mumtaz, A.; Hasanain, S. K.; Bertino, M. F. J. Magn. Magn. Mater. 2010, 322, 2199-2202.

28. Rastelli, A.; Ding, F.; Plumhof, J. D.; Kumar, S.; Trotta, R.; Deneke, C.; Malachias, A.; Atkinson, P.; Zallo, E.; Zander, T.; Herklotz, A.; Singh, R.; Křápek, V.; Schröter, J. R.; Kiravittaya, S.; Benyoucef, M.; Hafenbrak, R.; Jöns, K. D.; Thurmer, D. J.; Grimm, D.; Bester, G.; Dörr, K.; Michler, P.; Schmidt, O. G. Phys. Status Solidi B2012, 249, 687-696.

29. Kodama, R. H.; Berkowitz, A. E. Phys. Rev. B 1999, 59, 6321-6336.

30. Xiuli, Z.; Haisheng, X.; Yanni, Z. J. Phys. D: Appl. Phys. 2011, 44, 155501.

31. Li, X.; Wu, Y.; Chen, Z.; Wei, X.; Luo, H.; Dong, S. J. Appl. Phys. 2014, 115, 144103.

32. Masahiro, N.; Takefumi, K.; Daisuke, Y.; Koichi, S.; Yuya, N. Jpn. J. Appl. Phys. 2015, 54, 07HE15.

33. Herklotz, A.; Plumhof, J. D.; Rastelli, A.; Schmidt, O. G.; Schultz, L.; Dörr, K. J. Appl. Phys. 2010, 108, 094101.

34. Michler, P.; Kiraz, A.; Becher, C.; Schoenfeld, W. V.; Petroff, P. M.; Zhang, L.; Hu, E.; Imamoglu, A. Science 2000, 290, 2282-5.

35. Benson, O.; Santori, C.; Pelton, M.; Yamamoto, Y. Phys. Rev. Lett. 2000, 84, 2513-2516.

36. Zhang, Y.; Chen, Y.; Mietschke, M.; Zhang, L.; Yuan, F.; Abel, S.; Hühne, R.; Nielsch, K.; Fompeyrine, J.; Ding, F.; Schmidt, O. G. Nano Lett. 2016, 16, 5785-5791.

37. Chen, Y.; Zhang, J.; Zopf, M.; Jung, K.; Zhang, Y.; Keil, R.; Ding, F.; Schmidt, O. G. Nat. Commun. 2016, 7, 10387.

38. Pang, C.; Lee, G.-Y.; Kim, T.-i.; Kim, S. M.; Kim, H. N.; Ahn, S.-H.; Suh, K.-Y. Nat. Mater. 2012, 11, 795-801.

39. Zhao, D.; Katsouras, I.; Asadi, K.; Blom, P. W. M.; de Leeuw, D. M. Phys. Rev. B 2015, 92, 214115.

40. Tybell, T.; Paruch, P.; Giamarchi, T.; Triscone, J. M. Phys. Rev. Lett. 2002, 89, 097601.

41. Jo, J. Y.; Yang, S. M.; Kim, T. H.; Lee, H. N.; Yoon, J. G.; Park, S.; Jo, Y.; Jung, M. H.; Noh, T. W. Phys. Rev. Lett. 2009, 102, 045701.

42. Kleemann, W. Annu. Rev. Mater. Res. 2007, 37, 415-448.

43. Nattermann, T.; Pokrovsky, V.; Vinokur, V. M. Phys. Rev. Lett. 2001, 87, 197005.

44. Yang, S. M.; Jo, J. Y.; Kim, T. H.; Yoon, J. G.; Song, T. K.; Lee, H. N.; Marton, Z.; Park, S.; Jo, Y.; Noh, T. W. Phys. Rev. B 2010, 82, 174125.

45. Paruch, P.; Giamarchi, T.; Tybell, T.; Triscone, J.-M. J. Appl. Phys. 2006, 100, 051608.

46. Middleton, A. A. Phys. Rev. B 1992, 45, 9465-9468. 

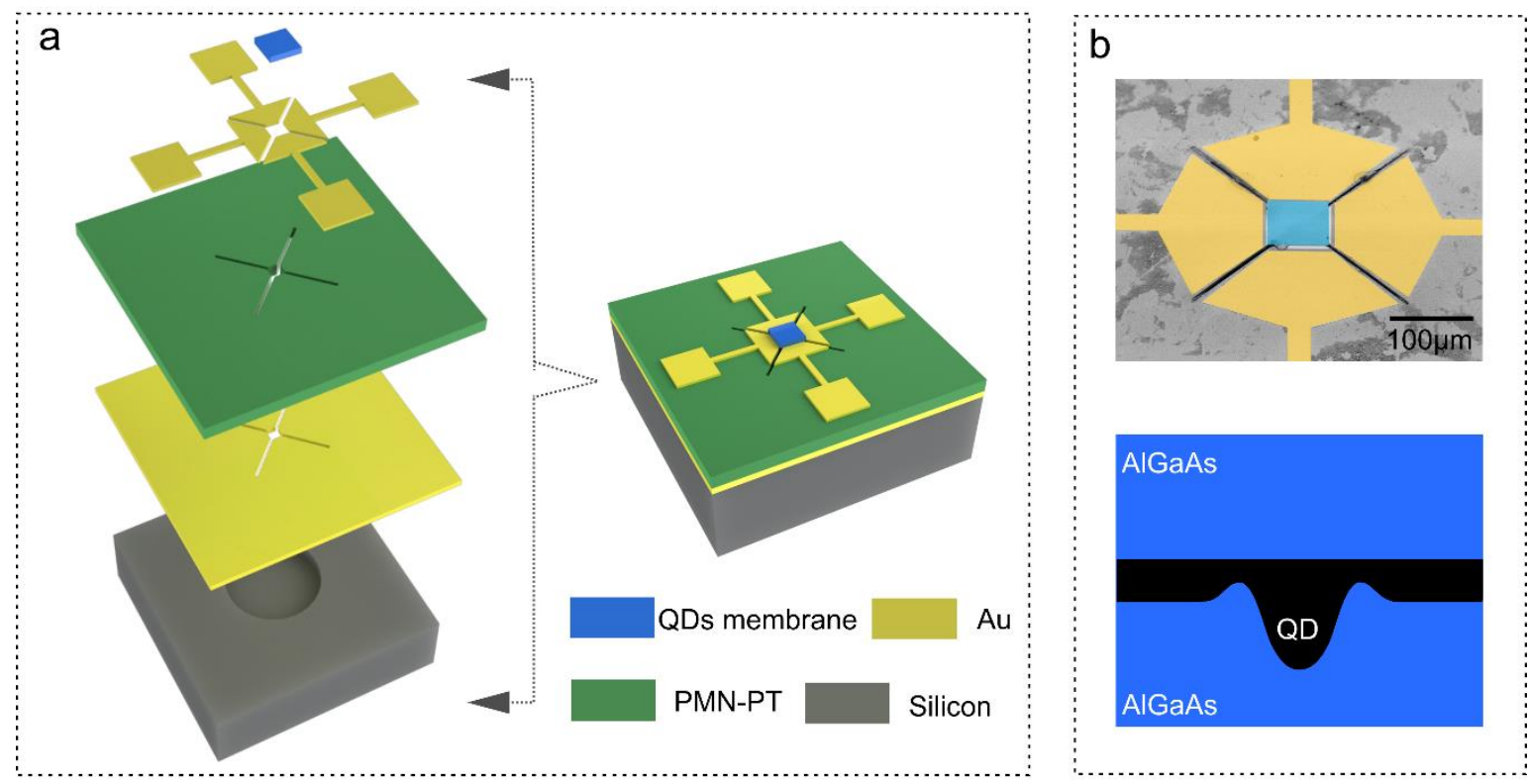

Figure 1 (a) Exploded view and sketch of the device. (b) Scanning electron microscope images of the device (false color). Lower panel is the sketch of a quantum dot embedded in an AlGaAs matrix. 

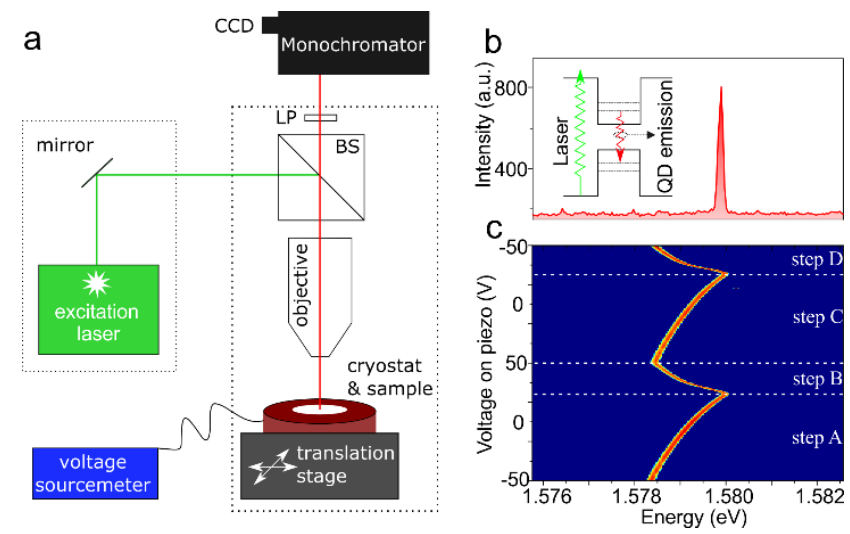

Figure 2 (a) Photo-luminescence measurement setup (BS: beam splitter; LP: long pass filter). (b) Typical spectrum of quantum dot emission. Inset shows a sketch of the energetic levels inside a quantum dot. (c) Spectral shift of the emission spectrum against the applied voltage. With continuously increasing electric field in the piezo, the emission first undergoes a blueshift (step A), followed by a red-shift (step B). 

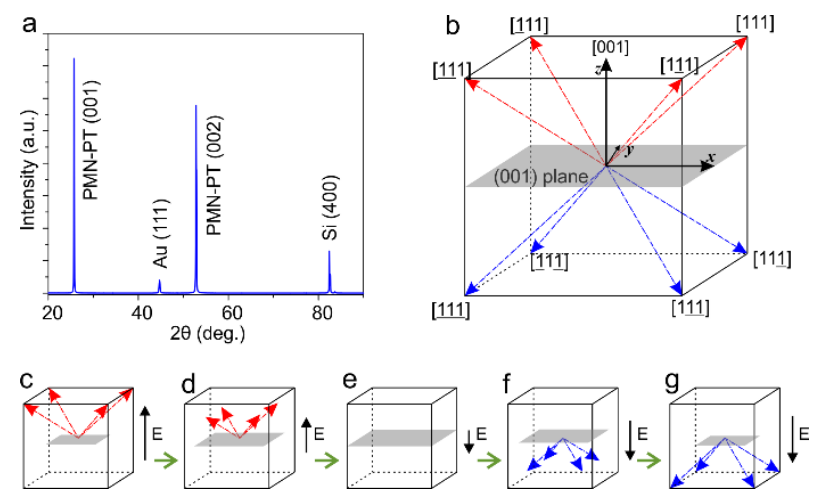

Figure 3 (a) X-ray diffraction of PMN-PT. Only (001) and (002) diffraction peaks appear, suggesting PMN-PT has a pure perovskite phase. As the piezo film is very thin, diffraction peaks from underlying $\mathrm{Au}$ and $\mathrm{Si}$ are also visible. (b) Rhombohedral unit cell with 8 possible polarization variants. (c-g) Schematic diagram of polarization configurations in [001] poled crystal, with grey plane indicate the amplitude of strain, under different voltages. (c) Fully poled crystal under large electric field leads to a net polarization along [001]. (d) As the electric field decreases, the net amount of polarization along [001] decreases as well. (e) Strain in (001) plane (grey) increases until the reversal field completely removes the polarization. In-plane strain is maximized at this point. (f, g) As the reversal field increases, the polarization flipped to the other direction and strain decreases. 

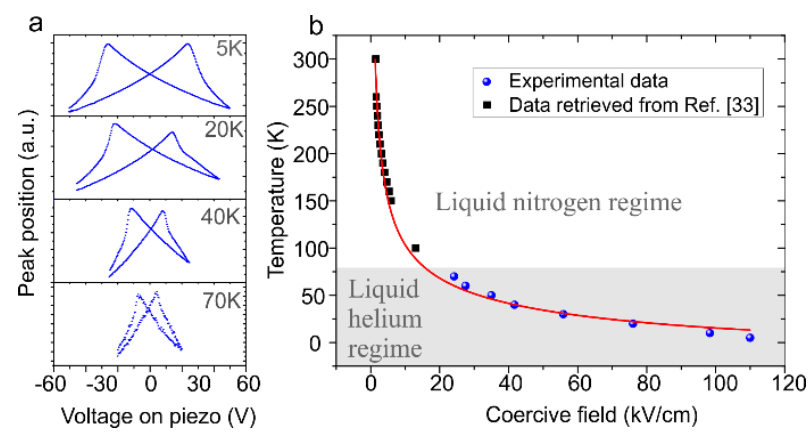

Figure 4 (a) Butterfly loop tuning behavior of a quantum dot emission peak at various temperatures, ranging from 5 Kelvin to 70 Kelvin. (b) Temperature-dependent coercive fields of PMN-PT, fitted by a theoretical model (red solid line). Coercive field decreases rapidly as temperature increases. 